\title{
Table-top X-ray Spectroscopy of Benzene Radical Cation
}

\section{Supporting information}

Michael Epshtein ${ }^{1,2}$, Valeriu Scutelnic ${ }^{1}$, Zheyue Yang $^{1}$, Tian Xue ${ }^{1}$, Marta L. Vidal ${ }^{3}$, Anna I. $\mathrm{Krylov}^{4}$, Sonia Coriani ${ }^{3}$, and Stephen R. Leone ${ }^{1,2,5^{*}}$

${ }^{1}$ Department of Chemistry, University of California, Berkeley, California 94720, United States

${ }^{2}$ Chemical Sciences Division, Lawrence Berkeley National Laboratory, Berkeley, California 94720, United States

${ }^{3}$ DTU Chemistry - Department of Chemistry, Technical University of Denmark, DK-2800, Kongens Lyngby, Denmark

${ }^{4}$ Department of Chemistry, University of Southern California, Los Angeles, California 90089, United States

${ }^{5}$ Department of Physics, University of California, Berkeley, California 94720, United States 


\section{X-ray transmission and absorption spectrum of neutral benzene}
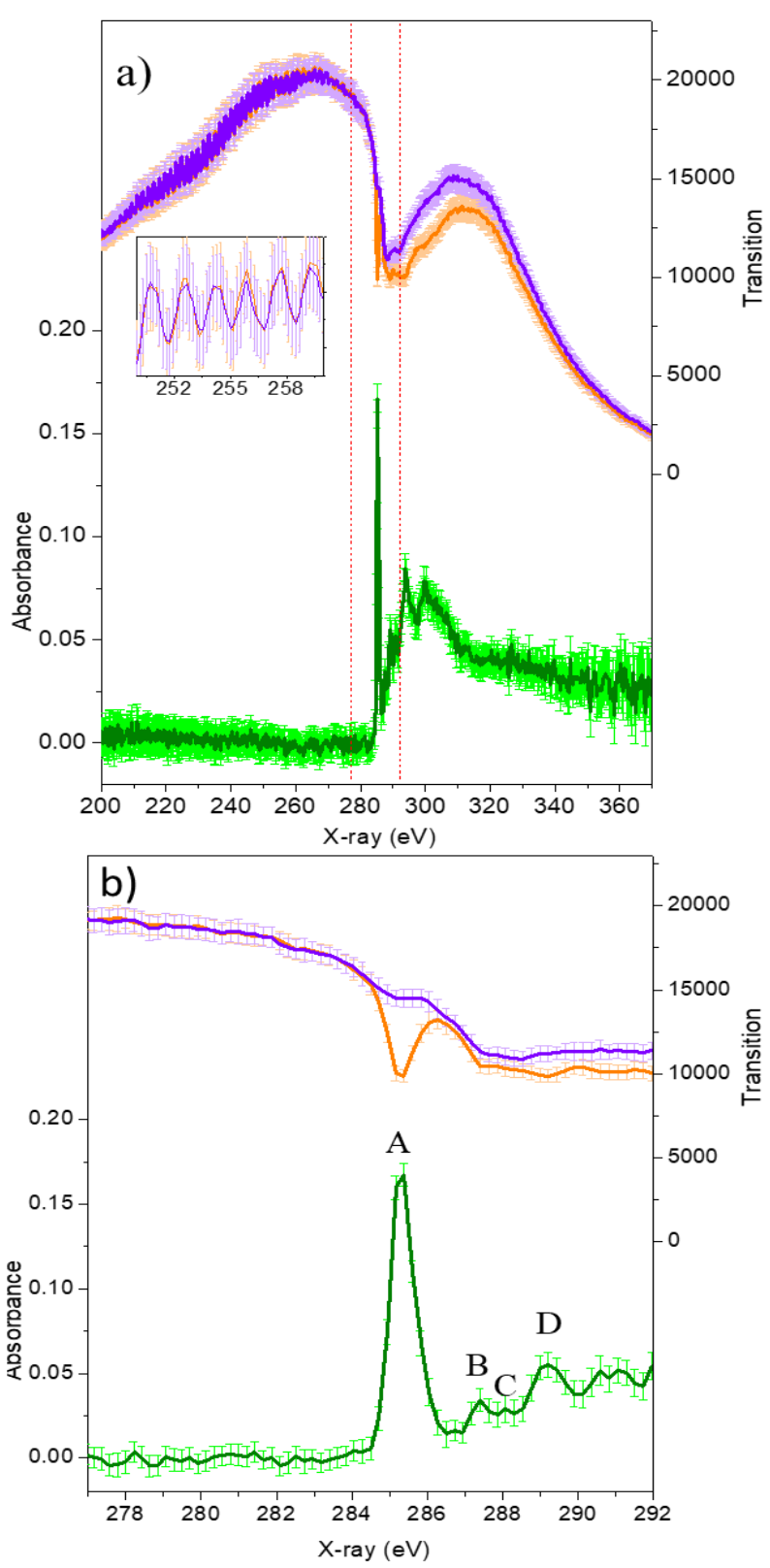

Figure S1. X-ray transmitted spectrum with and without benzene. (a) The purple line represents HHG soft X-ray radiation of the broadband probe pulse, $I_{\text {without Bz}}$, after passing through a $100 \mathrm{~nm}$ thick Al filter and two $100 \mathrm{~nm}$ thick Ti filters. The depletion in the $\mathrm{X}$-ray spectrum at $\sim 285 \mathrm{eV}$ is due to carbon contamination. The orange line represents X-ray transmitted spectrum after passing through a medium ( $1 \mathrm{~cm}$ length and pressure of less than $\sim 25$ Torr) of gas phase benzene, $I_{\text {with }}$ Bz. Each spectrum is an average of $128 \mathrm{CCD}$ images (1000 laser pulses per image). The spectra are obtained by vertical summation of the signal pixels detected at the camera. The green line represents the absorbance $(A)$ spectrum of neutral benzene, $A=\log \left(I_{\text {without Bz }} / I_{\text {with Bz }}\right)$. The region of interest related to transitions from carbon $\mathrm{K}$ edge to valence orbitals is marked with vertical dashed orange lines in panel (a) and presented in panel (b). The zoomed spectrum in the upper part of panel (a) shows the shape of the harmonics spectrum in the range of $250-260 \mathrm{eV}$, while in the range relevant to carbon K-edge the harmonics are broadened and produce a flat spectrum, as seen in panel (b). The error bars correspond to $95 \%$ confidence interval limits. 


\section{Calibration and spectral resolution of the experimental system}

The calibration of the spectrometer was performed by comparing recorded X-ray absorption spectra of argon and allyl radical with the previously reported transition energies, $;^{1,2}$ this is shown in Figure. S2.1 and S2.2.

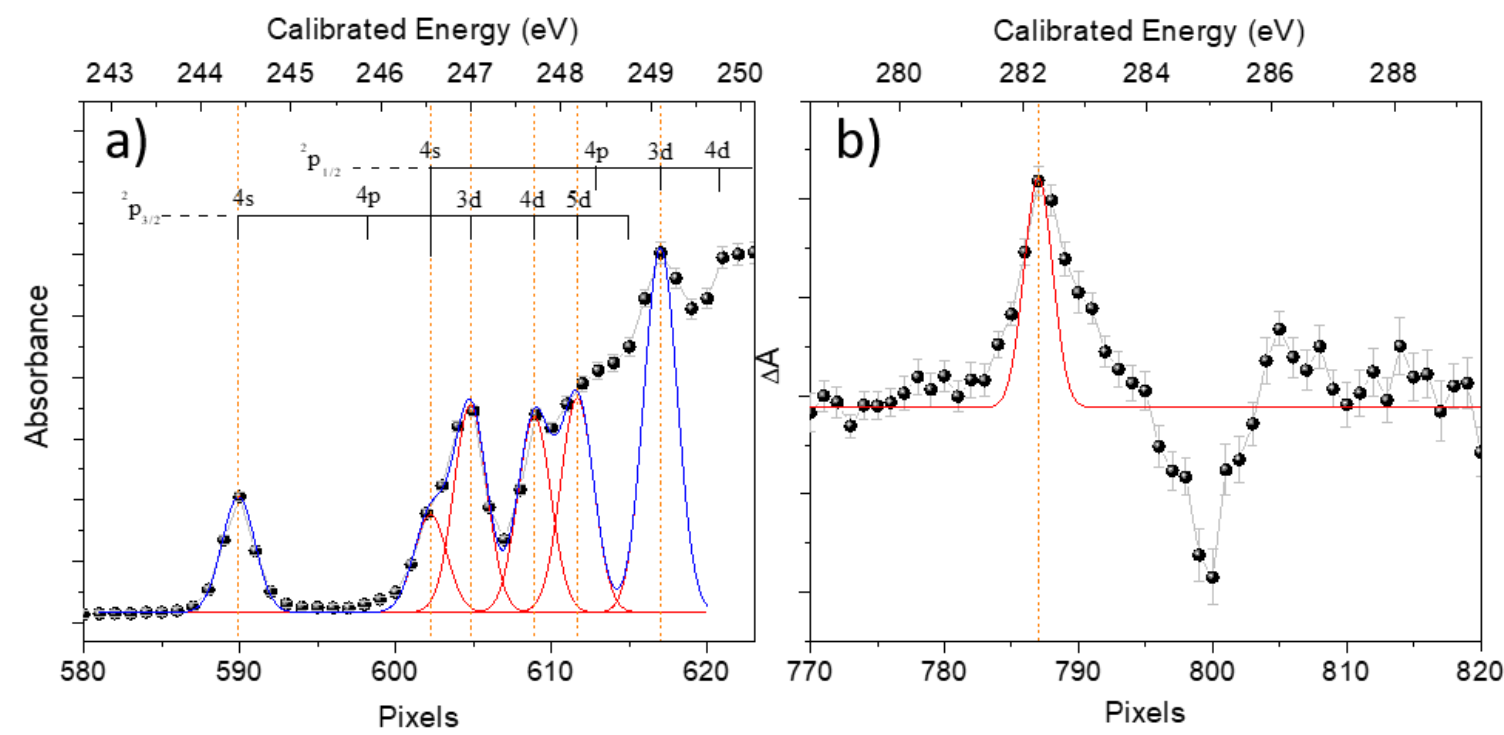

Figure S2.1. Calibration of the spectrometer. (a) The black circles connected by a gray line represent the absorption spectrum of argon, which shows the atomic core-Rydberg transitions used for calibration. The red line represents a Gaussian function fitted to the resonance transitions. The blue line corresponds to the sum of all six fitted transitions. (b) The black circles connected by a gray line represent the transient absorption spectrum of the allyl radical following $267 \mathrm{~nm}$ excitation promoting the radical to a repulsive electronic state. The red line represents a Gaussian function fitted to the known $1 \mathrm{~s}_{\mathrm{C}} \rightarrow \pi$ transition in the allyl radical, which is used as an additional point for calibration. Both spectra show the camera pixel numbers at the bottom of the figures, as observed by the camera, and the energy after calibration in the top of the panels. The gray error bars denote a $95 \%$ confidence limit. For a better calibration, the $1 \mathrm{~s}_{\mathrm{C}} \rightarrow \pi^{*}$ transition of neutral benzene is used as an additional point, since this transition is well known from previous studies.

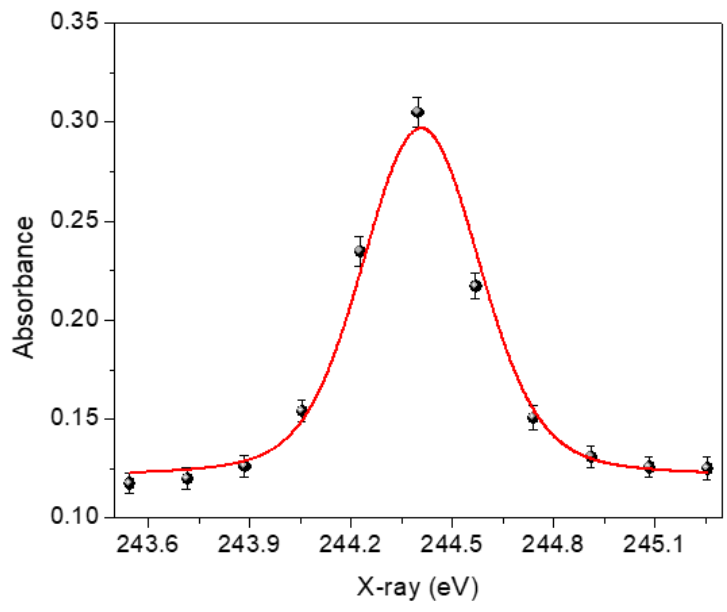


Figure S2.2. Spectral resolution. The peak represents the isolated ${ }^{2} \mathrm{p}_{3 / 2} \rightarrow 4 \mathrm{~s}$ transition in argon (taken from the calibrated spectrum in Figure. S2) fitted with a Voigt function with a Lorentzian (width of $0.114 \mathrm{eV}$ corresponding to the lifetime of ${ }^{2} \mathrm{p}_{3 / 2}$ core hole state ${ }^{1}$ ) and a Gaussian (width of $0.35 \pm 0.03 \mathrm{eV}$, corresponding to the spectral resolution of the system).

The difficulties in resolving the experimental spectrum with regard to vibrational resolution come from the peak shapes, peak overlaps due to energetically close transitions, and peak broadening, which is caused by several reasons. In the present experiment, the peak broadening appears to be due to a very short lifetime of the core levels, causing a Lorentzian broadening of about $0.1 \mathrm{eV}$, and a Gaussian contribution due to the instrument resolution. In addition, the peaks show vibrational broadening. In the static spectrum, the vibrational structure of the $1 \mathrm{~s} C \rightarrow \pi^{*}$ transition has been resolved and the vibrational broadening of other electronic transitions has been discussed. ${ }^{3,4}$ However, the vibrational structure of the X-ray spectrum of the cations is unknown and would be difficult to resolve due to the complex vibronic manifold arising from the two Jahn-Teller potential energy surfaces (PESs), due to internal vibrational energy in the room temperature benzene neutral and because the vibrational states of the ion depend on the FC factors for the two-photon ionization from each neutral vibrational state. To estimate the spectral resolution, we deconvoluted the argon peak at $244.4 \mathrm{eV}$ and found the best fit with a Voigt function with a Lorentzian width of $0.114 \mathrm{eV}$ and a Gaussian width of $0.35 \pm$ $0.03 \mathrm{eV}$, shown in Figure. S3. The Gaussian contribution comes from the grating efficiency and camera resolution. Our UV beam has a bandwidth of $400 \mathrm{~cm}^{-1}$, which results in some vibrational excitation of benzene cations, rendering vibrational analysis of the spectra difficult. For all mentioned reasons, we do not attempt to resolve the vibrational structure of the X-ray spectra. 


\section{Initial population of vibrational states at room temperature}

One of the most important parts of the presented experiment was to find the best experimental conditions for two photon ionization, and in this way to obtain relatively cold benzene cation. Two photon ionization with $267 \mathrm{~nm}$ is in resonance with the ionization region and prepares the cation with a relatively small amount of vibrational energy as discussed in the main text, but still close to the vibrationless ground state cation. The temperature $\left(\sim 21 \mathrm{C}^{\circ}\right)$ influences the prepared cation due to initial vibrational population of the neutral benzene with respect to a Boltzmann distribution. Figure. S3 presents the population fraction as function of the vibrational energy.

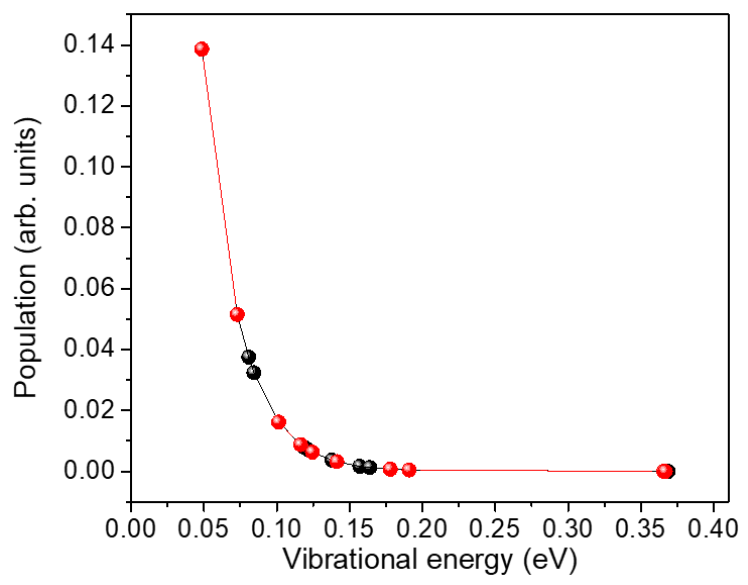

Figure S3. The population of vibrational neutral benzene due to room temperature thermal energy. The red and black circles represent the energy of the vibrational fundamental modes and their related population according to Boltzmann distribution. The red circles are related to modes degenerate in energy (two different motions for one energy due to the high symmetry of benzene). Different combinations and overtones are not taken into account because the lowest overtone is $0.108 \mathrm{eV}$, and it has a very small impact on the population, and all the others have much less. (the lowest vibrational state in neutral benzene is $0.049 \mathrm{eV}\left(405 \mathrm{~cm}^{-1}\right)$ ). The vibrational energies are taken from Ref. 5 from this distribution, the vibrational ground-state population is about 45\%; all the other molecules are distributed among different vibrational states. From this data, the estimated average thermal vibrational energy is about $0.05 \mathrm{eV}$. 


\section{Pressure-dependence study}

Special care was taken to obtain an unsaturated absorbance spectrum of neutral benzene. The high vapor pressure of benzene ( 100 Torr), high absorption cross section at the carbon Kedge, and low intensity of the X-ray radiation, make it easy to reach the saturated regime of the small number of X-ray photons per camera pixel leading to an asymmetric broadening (on the high-energy side) of the main peak (as seen in Figure. S4(c)) due to the saturated vibrational structure and a different ratio between the main peak relative to the lower peaks and to the core ionization region. The spectra represented by light blue and blue lines are below the saturated regime, because the ratio between the main peak and the other peaks and to the ionization region is nearly constant at different pressures. In addition, the spectrum is very similar to the spectrum reported in Refs. 3,4. By comparing the signal $(\sim 300 \mathrm{eV})$ at highest pressure ( 100 torr) with signal at unsaturated regime (black line and blue line in Figure. S4(a)), we estimate the unsaturated linear regime to be when the pressure in the cell is lower than 25 Torr.

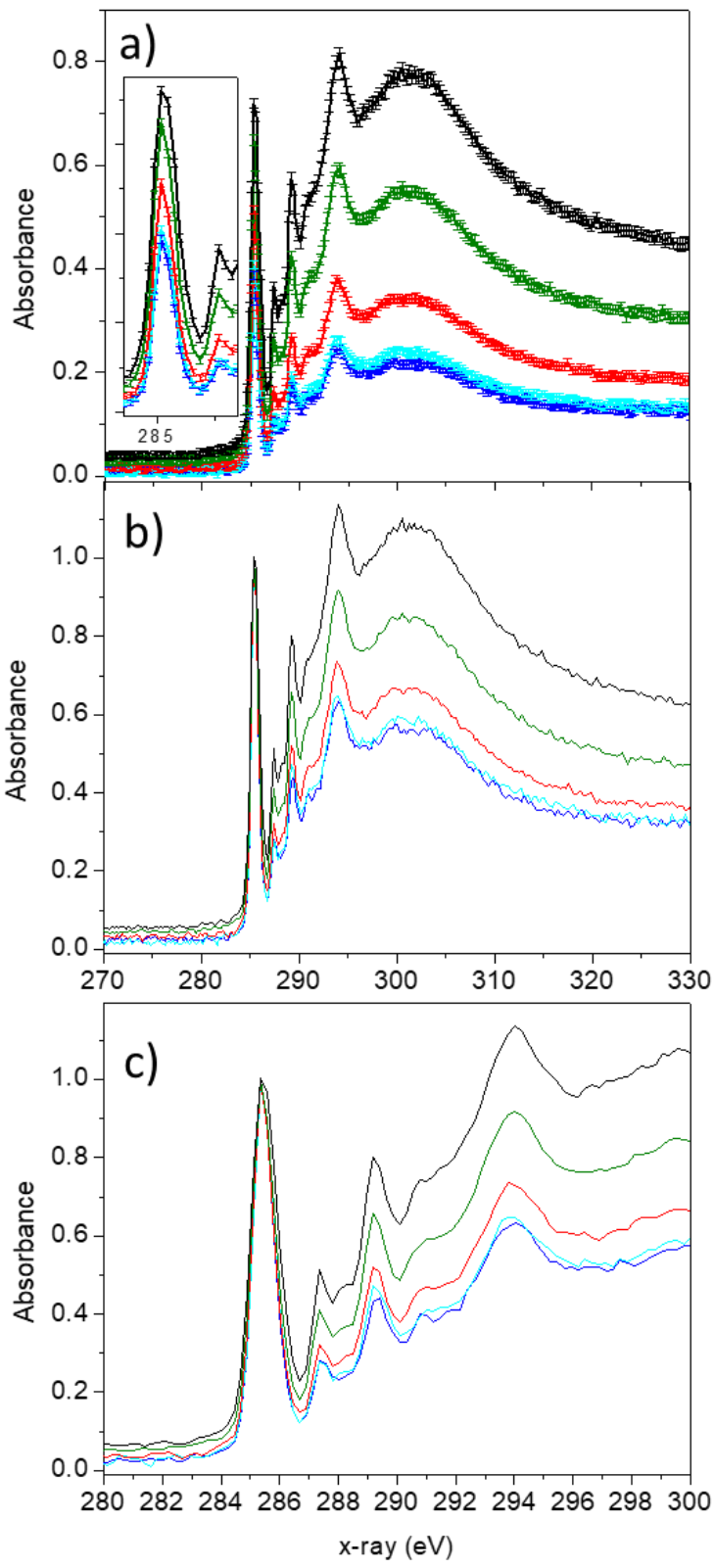

Figure S4: (a) NEXAFS spectra of ground state neutral benzene at different pressures; black, green, red, light blue, and dark blue lines represent the absorbance signal at different pressures of benzene in the interaction region with the X-ray radiation, from highest to lowest pressure, respectively. Each spectrum is an average of 128 CCD images (1000 laser pulses per image). The pressure in the interaction region is controlled by a valve located between the cell and the source. The source is liquid benzene at room temperature with a vapor pressure of 100 Torr. Note that the pressure in the cell is lower than 100 Torr even when the valve is fully open, since the molecules escape through two holes (200 microns diameter) made for the X-ray and UV passage through the cell. The left panel is the zoomed main peak. (b) Normalized data (shown in panel (a)) with respect to the main peak at $285 \mathrm{eV}$. (c) Zoomed spectrum in the 280 - $300 \mathrm{eV}$ region, relevant to this work. 


\section{Add-back correction for transient spectra}

The differential absorption spectrum of the cations is calculated as $\Delta A=\log \left(I_{\text {without }}\right.$ $\mathrm{UV} / I_{\text {with UV }}$ ). The transmitted signal without UV ( $I_{\text {without UV }}$ ) is due to neutral benzene alone, while the transmitted signal with UV ( $I_{\text {with UV }}$ ) is due to the neutral and ionized benzene. Therefore, the number of neutral molecules in the sample is different for experiments with UV and without UV and depends on the number of cations. By adding back a scaled static spectrum only of neutral benzene molecules, it is possible to obtain a pure cation signal. The main uncertainty of this procedure is how to correctly scale the static spectrum based on the percentage of the ionized molecules in the interaction region. The unknown two-photon ionization cross section further complicates the estimation of the percentage of the ionized molecules. We used several estimates to set the limit on the cation fraction. First, we assumed that the cross section for core ionization of neutral benzene is similar to that of benzene cation. Second, we assumed that the total intensity of peaks $\mathrm{F}$ and $\mathrm{G}$ should be roughly equal to the intensity of peak $\mathrm{A}$, because these peaks are due to $1 \mathrm{sc}_{\mathrm{C}} \rightarrow \pi^{*}$ transitions. Thus, the ratio of peak $\mathrm{A}$ and the signal in the core ionization region (i.e., above $292 \mathrm{eV}$ in Figure. S5), should be similar to the ratio between peak F and the ionization region. This is achieved by adding $2 \%$ of the static neutral spectrum to the transient spectrum. By adding $2 \%$ of the static neutral spectrum, the depleted signal completely disappears, which is an indication that our assumptions are close to the correct value. We note that the intensity of features below $284 \mathrm{eV}$ (i.e., peak E) are not affected by the "add back" procedure. It is important to note that the percentage can easily change from experiment to experiment due to the ionization efficiency (different focusing condition of the UV beam, beam shape and intensity, intersection between UV and X-ray flux in the sample cell) and therefore different percentages were used in Figure. S6. 

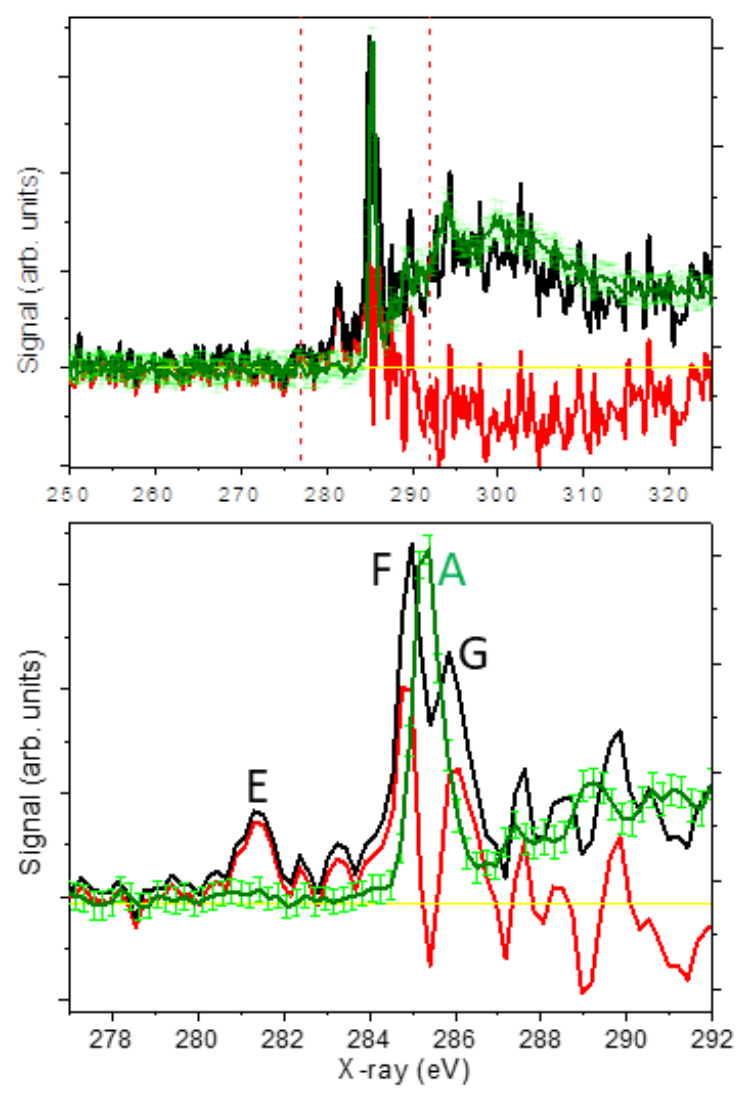

Figure S5. The transient X-ray spectrum with and without added scaled static spectrum. The red line in panels (a)-(b) is the transient spectrum of the cation before the add back correction, taken at 0-50 $\pm 50 \mathrm{fs}$ delay between the pump (UV) and probe (X-ray) beam. The black line in panels (a) and (b) is the transient spectrum after adding $2 \%$ of the static spectrum. The green line in each panel shows the static spectrum (ground-state spectrum of the neutral benzene). Panel (b) is a zoom of panel (a). 


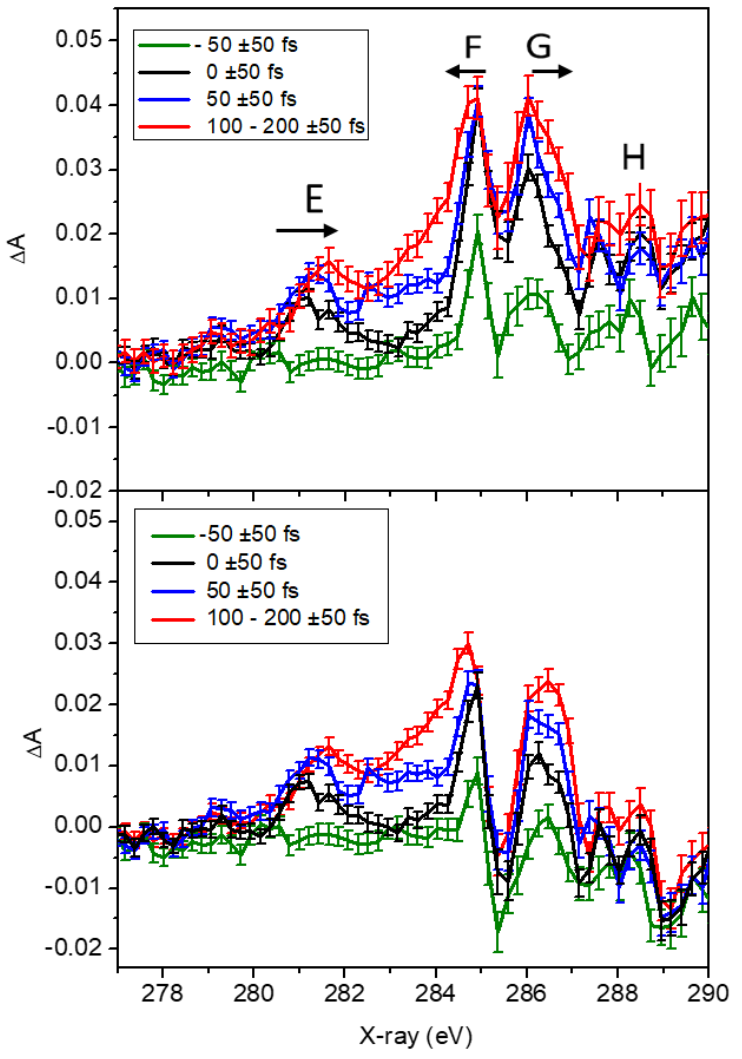

Figure S6. Transient X-ray absorption spectra measured at different time delays between the pump and probe beams. The lower panel is the raw data without added scaled static spectrum and the upper panel is after adding $3 \%$ of the static spectra to the spectra represented by black, blue and red lines, and $2 \%$ to the spectrum represented by a green line, since just a small part of the UV beam interacts with the X-ray beam at the negative time delay (green) of $-50 \pm 50$ fs delay between the pump (UV) and probe (X-ray) beam. Black line represents $0 \pm 50$ fs delay where the probe and pump beam fully overlap in time. The blue line represents $50 \pm 50 \mathrm{fs}$ and the red line represents a range of $100-200 \pm 50$ fs delay. 


\section{REFERENCES}

${ }^{1}$ King, G. C.; Tronc, M.; Read, F. H.; Bradford, R. C. An investigation of the structure near the $\mathrm{L}_{2,3}$ edges of argon, the $\mathrm{M}_{4,5}$ edges of krypton and the $\mathrm{N}_{4,5}$ edges of xenon, using electron impact with high resolution. J. Phys. B. 1997, 10, 2479.

${ }^{2}$ Alagia, M.; Bodo, E.; Decleva, P.; Falcinelli, S.; Ponzi, A.; Richter, R.; Stranges, S. The soft X-ray absorption spectrum of the allyl free radical. Phys. Chem. Chem. Phys. 2013, 15, 1310.

${ }^{3}$ Ma, Y.; Sette, F.; Meigs, G.; Modesti, S.; Chen, C. T. Breaking of ground-state symmetry in coreexcited ethylene and benzene. Phys. Rev. Lett, 1989, 63, 2044.

${ }^{4}$ Rennie, E. E. Kempgens, B. Koppe, H. M.; Hergenhahn, U.; Feldhaus, J.Itchkawitz, B. S.; Kilcoyne, A. L. D.; Kivimaki, A.; Maier, K.; Piancastelli, M. N.; Polcik, M.; Rudel, A.; Bradshaw, A. M. A comprehensive photoabsorption, photoionization, and shake-up excitation study of the $\mathrm{C} 1 \mathrm{~s}$ cross section of benzene. J. Chem. Phys. 2000, 113, 7362-7375.

${ }^{5}$ Gardner, A. M.; Wright, T. G. Consistent assignment of the vibrations of monosubstituted benzenes. $J$. Chem. Phys. 2011, 135, 114305. 